Adriana LuCiana Moreno Camargo ${ }^{1}$

Gisela Rosa Franco Salerno ${ }^{1}$

Regina Célia Teixeira Gomes²

Ricardo SANTOS SimÕes ${ }^{3}$

Rodrigo de Aquino CASTRO ${ }^{4}$

Manoel joão Batista Castello Girão ${ }^{4}$

Artigo Original

Palavras-chave

Terapia por estimulação elétrica Diafragma da pelve/anatomia \& histologia

Uretra

Ratos

Keywords

Electric stimulation therapy Pelvic floor/anatomy \& histology

Urethra

Rats

Adriana Luciana Moreno Camargo Departamento de Ginecologia da Escola Paulista de Medicina Universidade Federal de São Paulo - UNIFESP Rua Borges Lagoa, 783, conjunto $31,3^{\circ}$ anda (EP: 04038-031

São Paulo (SP), Brasi

Recebido

10/02/2013

Aceito com modificacoones

$26 / 03 / 2013$

\section{Aspectos morfológicos da uretra de ratas após eletroestimulação do assoalho pélvico}

\author{
Morphological aspects of the urethra in female rats after electrical \\ stimulation of the pelvic floor
}

\section{Resumo}

OBJETIVO: Avaliar os efeitos da eletroestimulação (ES) do assoalho pélvico na uretra de ratas. MÉTODOS: Quarenta ratas adultas foram distribuídas, randomicamente, em quatro grupos com dez animais cada: Ctrl - sem intervenção; Sham - não foi submetido a ES, recebeu um eletrodo dentro da vagina; Exp6 - submetido a seis sessões de ES do assoalho pélvico; e Exp 12 - submetido a 12 sessões de ES do assoalho pélvico. Ao final do experimento, todos os animais foram anestesiados, e o terço médio da uretra foi retirado, fixado em líquido de Bovin e processado para estudo histomorfométrico. Alguns cortes foram corados pela hematoxilina e eosina, para descrição morfológica e morfométrica, e outros, pelo picrosirius red, para avaliação do colágeno total. As espessuras da camada muscular e do epitélio foram obtidas, nos 4 quadrantes da uretra, pela realização de 20 medições em cada animal. O número de vasos sanguíneos presentes na lâmina própria foi obtido nos quatro quadrantes, em uma área de $10^{3} \mathrm{~mm}^{2}$ por quadrante, sendo as imagens obtidas pelo programa de análise de imagens AxioVision ${ }^{\circledR}$ REL 4.3 (Carl Zeiss). A proporção de colágeno e de fibras musculares foi obtida de duas imagens por quadrante, de cada lâmina da uretra corada pelo picrosirius red, com auxílio do programa lmagelab ${ }^{\circledR}$. Os dados foram submetidos à análise de variância (ANOVA) e ao teste de comparações múltiplas de Tukey-Kramer $(p<0,05)$. RESULTADOS: A morfometria do colágeno, número de vasos sanguíneos e espessura do epitélio não mostraram alterações significantes. No entanto, a espessura do tecido muscular periuretral mostrou aumento significante no grupo Exp 12 em relação aos outros grupos (Exp 12* $>$ Exp6==Ctrl==Sham; $\left.{ }^{*} p<0,05\right)$. CONCLUSÃO: A eletroestimulação funcional prolongada do assoalho pélvico induziu aumento na espessura da camada muscular periuretral em ratas.

\section{Abstract}

PURPOSE: To evaluate the effects of electrical stimulation (ES) of the pelvic floor on the urethra of female rats. METHODS: Forty adult rats were divided at random into four groups of ten animals each: Ctrl - without intervention; Sham - not submitted to ES, but with an electrode inserted into the vagina; Exp6 - submitted to six sessions of ES of the pelvic floor, and Expl 2 - submitted to 12 sessions of ES of the pelvic floor. At the end of the experiment, all animals were anesthetized and the middle third of the urethra was removed, fixed in Bouin's fluid and processed for histomorphometric study. Sections were stained with hematoxylin and eosin for morphological and morphometric description, and others were stained with picrosirius red for the quantitation of total collagen. The thicknesses of the muscle layer and of the epithelium were determined, in 4 quadrants of the urethra, by performing 20 measurements per animal. The number of blood vessels present in the lamina propria was counted in the four quadrants over an area of $10^{3} \mathrm{um}^{2}$ per quadrant and the images were obtained using the image analysis program AxioVision ${ }^{\circledR}$ REL 4.3 (Carl Zeiss). The collagen and muscle fiber ratios in the urethrae were calculated from two images per quadrant of every slice stained with picrosirius red, employing the Imagelab ${ }^{\circledR}$ Program. Data were subjected to analysis of variance (ANOVA) and the Tukey-Kramer multiple comparison test $(p<0.05)$. RESULTS: The morphometry of the collagen, number of blood vessels and thickness of the epithelium showed no significant changes; however, the thickness of the periurethral muscle tissue increased significantly in Exp 12 group, compared to the other groups (Exp 12* $>$ Exp6 $==$ Ctrl $==$ Sham; $\left.{ }^{*} p<0.05\right)$. CONCLUSION: Prolonged functional electric stimulation of the pelvic floor induced an increase in periurethral muscle thickness in rats.

\section{Trabalho realizado no Departamento de Ginecologia da Escola Paulista de Medicina, Universidade Federal de São Paulo - UNIFESP -} São Paulo (SP), Brasil.

Programa de Pós-graduação em Ginecologia da Escola Paulista de Medicina, Universidade Federal de São Paulo - UNIFESP - São Paulo (SP), Brasil.

2Programa de Pós-doutorado do Departamento de Ginecologia da Escola Paulista de Medicina, Universidade Federal de São Paulo UNIFESP - São Paulo (SP), Brasil.

${ }^{3}$ Hospital Universitário, Universidade de São Paulo - USP - São Paulo (SP), Brasil.

4Departamento de Ginecologia da Escola Paulista de Medicina, Universidade Federal de São Paulo - UNIFESP - São Paulo (SP), Brasil. 


\section{Introdução}

A incontinência urinária é condição muito prevalente, com significativos aspectos médicos e sociais, constituindo importante problema de saúde pública e apresentando grande impacto na qualidade de vida. Em condições normais, a incontinência urinária é mais frequente na população feminina, atingindo aproximadamente $10 \%$ da população geral e mais de $30 \%$ nas mulheres com mais de 60 anos. Vários fatores contribuem para a incontinência urinária, dentre os quais se destacam a topografia intra-abdominal do colo vesical, os componentes muscular, vascular e a elasticidade passiva da parede uretral, assim como a musculatura e a inervação adequada do assoalho pélvico ${ }^{1,2}$.

A Sociedade Internacional de Continência (ICS) refere que o tratamento das diferentes formas de incontinência urinária deve se iniciar sempre pelos procedimentos menos invasivos, sendo o fortalecimento muscular do assolalho pélvico a primeira escolha, por intermédio dos exercícios perineais, dos cones vaginais e da $\mathrm{ES}^{3}$. A escolha para o tratamento conservador também é favorecida pelo fato de o tratamento cirúrgico não ser eficaz em todos os casos. Recidivas dos sintomas podem ocorrer dentro de cinco anos após o procedimento cirúrgico ${ }^{4}$.

Assim, a reabilitação do assoalho pélvico é prescrita como tratamento de primeira linha para mulheres com incontinência urinária de esforço, particularmente em casos de incontinência urinária sem prolapso uterino de primeiro grau. Entre os tratamentos conservadores, as investigações indicam que os exercícios perineais, os cones vaginais e a eletroestimulação são igualmente efetivos $^{5,6}$.

Poucos são os trabalhos que abordam temas como a ES, uretra e incontinência urinária. Acredita-se que o estímulo elétrico seja capaz de aumentar a pressão intrauretral por meio da estimulação direta dos nervos eferentes para a musculatura, com aumento do fluxo sanguíneo para os músculos do assoalho pélvico, o que restabelece as conexões neuromusculares, com melhora da função das fibras musculares, hipertrofiando-as e modificando o seu padrão de ação ${ }^{7}$.

Assim, a escassez de estudos histológicos da uretra com o uso da estimulação elétrica motivou a realização do presente estudo.

\section{Métodos}

Trata-se de estudo experimental prospectivo, realizado na Disciplina de Uroginecologia e Cirurgia Vaginal do Departamento de Ginecologia e na Disciplina de Histologia e Biologia Estrutural do Departamento de Morfologia da
Universidade Federal de São Paulo (UNIFESP-EPM). Foram utilizadas 40 ratas (Rattus norvegicus albinus) adultas, virgens, pesando aproximadamente $250 \mathrm{~g}$, fornecidas pelo Centro de Desenvolvimento de Modelos de Experimentação (CEDEME) da UNIFESP-EPM. Este estudo foi aprovado pelo Comitê de Ética em Pesquisa da referida instituição (parecer $n^{\circ}$ 088/03) e seguiu as diretrizes do Conselho Canadense para os Cuidados dos Animais.

Os animais foram alojados ( 5 por gaiola) em Biotério, com luz (12/12 - ciclo claro/escuro) e temperatura controladas $\left(23-25^{\circ} \mathrm{C}\right)$, alimentados com ração peletizada para ratos (Labina-Purina, São Paulo, Brasil) e água ad libitum. Depois de duas semanas, período de adaptação, as ratas foram divididas em quatro grupos, a saber: Ctrl $(\mathrm{n}=10)$ - animais que não receberam estimulação elétrica; Sham $(n=10)$ - animais que não receberam estimulação elétrica, mas que reberam eletrodo endovaginal; $\operatorname{Exp} 6(\mathrm{n}=10)$ - animais submetidos a 6 sessões de estimulação elétrica endovaginal; Exp12 ( $\mathrm{n}=10)$ - animais submetidos a 12 sessões de estimulação elétrica endovaginal.

As sessões de ES do assoalho pélvico foram realizadas com uso de um estimulador específico, equipamento de fabricação nacional da marca Viotti (Viotti S/A, São Paulo, Brasil), com sonda especialmente desenvolvida para o estudo, com $2 \mathrm{~mm}$ de diâmetro. Foi utilizada corrente de baixa frequência no modo pulso-disparo, com parâmetros convencionados em frequência de $50 \mathrm{~Hz}$, com pulso de $1 \mathrm{~ms}$ e intensidade de $20 \mathrm{~mA}$. Os animais foram submetidos a 2 sessões semanais, com duração de 15 minutos cada, durante 3 (Exp6) ou 6 (Exp12) semanas. Antes de cada sessão, as ratas foram anestesiadas com xilazina $(15 \mathrm{mg} / \mathrm{kg})$ e cetamina $(30 \mathrm{mg} / \mathrm{kg})$, diluídas em solução fisiológica $(0,9 \%)$ e aplicadas por via subcutânea.

Após oito dias do término das sessões, os animais foram novamente anestesiados e, na sequência, por incisão longitudinal no abdome, foram retiradas, em bloco, a bexiga e a uretra. Estas foram distendidas sobre papel cartão e imediatamente mergulhadas em líquido de Bouin para fixação. Após um período de 24 horas, as peças foram dissecadas e separadas em duas regiões distintas e macroscopicamente delimitadas: bexiga e uretra. Neste estudo, seccionamos transversalmente o terço médio da uretra, o qual foi desidratado em álcool etílico em concentrações crescentes, diafanizado pelo xilol e impregnado por parafina líquida. Os fragmentos foram incluídos e orientados de tal maneira que pudéssemos obter cortes transversais perpendiculares ao maior eixo da uretra. Cortes de $5 \mathrm{~mm}$ foram obtidos em micrótomo do tipo Minot e, posteriormente, as lâminas coradas pela hematoxilina-eosina e pelo picrosirius red. 
Para efetuar as medidas das espessuras da camada muscular e do epitélio, a uretra foi inicialmente dividida em 4 quadrantes, tendo-se obtido a média de 20 mensurações em cada animal e tendo sido efetuadas 5 medições em cada um dos quadrantes. O número de vasos sanguíneos na lâmina própria foi contado também nos quatro quadrantes, numa área de $1.000 \mathrm{~mm}^{2}$ por quadrante, realizando-se a média desses valores. Essas imagens foram obtidas e analisadas utilizando-se uma câmera de alta resolução (AxioCam Mrc - Carl Zeiss) acoplada a microscópio de luz (objetiva de 40X) e programa de análise de imagens AxioVision ${ }^{\circledR}$ REL 4.3 da Carl Zeiss.

Para a análise morfométrica das fibras colágenas e musculares, empregou-se a técnica de representação de imagem, efetuando-se a quantificação por meio de sistema computadorizado, constituído por microscópio de luz (Carl Zeiss) com objetiva 40X, câmera de vídeo colorida (Sony), computador Pentium 4, IBM, placa gráfica para aquisição de imagem 640x480 pixels (correspondente, em micrômetros, à área de $21,23 \times 10^{3} \mathrm{~mm}^{2}$ ), 24 bits (16 milhões de cores) e software de processamento e análise de imagem Imagelab ${ }^{\circledR}$ (Softium Informática Ltda.). Por meio desse software, foram selecionados quatro

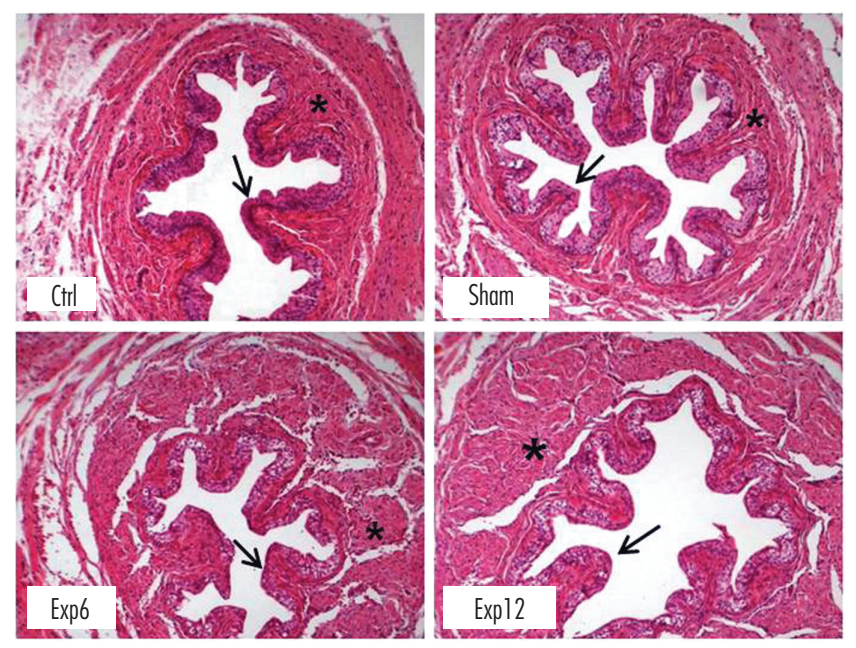

Figura 1. Fotomicrogafias de cortes transversais da uretra de ratas pertencentes aos quatro grupos de estudo. Nota-se a presença de pregas (setas) fazendo saliência para o lúmen uretral e camada de fibras musculares lisas no sentido longitudinal (*). H.E 400X. quadrantes da lâmina da uretra, avaliando-se a média da porcentagem encontrada.

Os dados obtidos foram submetidos à análise de variância (ANOVA) complementada pelo teste de contraste de Tukey-Kramer. Fixou-se em 5\% (p<0,05) o nível para rejeição da hipótese de nulidade, assinalando-se com asterisco os valores significantes.

\section{Resultados}

\section{Morfologia}

A uretra, nos vários grupos estudados, apresenta basicamente a mesma estrutura, ou seja, encontra-se formada por duas camadas bem distintas: mucosa e muscular. A mucosa apresenta pregas longitudinais e está revestida internamente por um epitélio de transição (pseudoestratificado), que repousa sobre uma lâmina própria formada por tecido conjuntivo frouxo, rico em vasos sanguíneos. Ao redor da lâmina própria, porção externa, podemos identificar a camada muscular lisa, constituída internamente por feixes longitudinais e, externamente, por feixes circulares. Nos grupos Ctrl e Sham, os aspectos morfológicos são semelhantes, ou seja, notam-se as duas camadas bem definidas. No entanto, as fibras musculares longitudinais são mais esparsas (Figura 1). Já nos grupos Exp6 e Exp12, os feixes de fibras musculares longitudinais encontram-se mais agrupados (Figura 1).

\section{Morfometria}

$\mathrm{Na}$ Tabela 1, estão expressos os dados das espessuras do epitélio periuretral e da camada muscular, assim como a contagem das fibras colágenas e o número de vasos da lâmina própria nos quatro grupos estudados. Com relação ao epitélio periureteral, na contagem de fibras colágenas e no número de vasos sanguíneos, não foram observadas diferenças significantes entre os vários grupos de estudo. No entanto, a espessura da camada muscular mostrou-se significativamente maior no grupo Exp12 em relação aos grupos Exp6, Ctrl e Sham (Exp12=329,8 $\pm 73,7 *>\operatorname{Exp} 6$ $=272,2 \pm 65,6>\operatorname{Ctrl}=256,4 \pm 41,7=$ Sham $=273,2 \pm 59,3$; $* \mathrm{p}<0,05)$.

Tabela 1. Médias e desvios-padrão do epitélio superficial, dos vasos sanguíneos e do colágeno total, presentes na camada muscular da uretra de ratas nos quatro grupos estudados

\begin{tabular}{|c|c|c|c|c|}
\hline & \multicolumn{4}{|c|}{ Grupos } \\
\hline & Ctrl & Sham & Exp6 & Expl2 \\
\hline Epitélio (mm) & $34,2 \pm 5,4$ & $34,2 \pm 5,4$ & $35,2 \pm 7,8$ & $34,6 \pm 7,0$ \\
\hline $\operatorname{Vasos}\left(10^{3} \mu \mathrm{m}^{2}\right)$ & $35,5 \pm 32,6$ & $35,2 \pm 99,1$ & $35,6 \pm 18,0$ & $35,4 \pm 14,6$ \\
\hline Músculo (mm) & $256,4 \pm 41,7$ & $273,2 \pm 59,3$ & $272,2 \pm 65,6$ & $329,8 \pm 73,7^{\star}$ \\
\hline Colágeno (\%) & $51,1 \pm 4,9$ & $52,2 \pm 3,7$ & $48,6 \pm 6,3$ & $50,9 \pm 5,1$ \\
\hline
\end{tabular}

Ctrl: controle; Sham: não recebeu estímulo, mas o eletrodo foi introduzido; Exp6: recebeu 6 estímulos; Exp 12: recebeu 12 estímulos. (Exp 12>Exp6==Ctrl==Sham; $\left.{ }^{*} p<0,05\right)$. 


\section{Discussão}

O mecanismo da continência urinária e a função desempenhada pelas estruturas uretrais são muito complexos. Entretanto, é conhecido que fatores como gravidez e hipoestrogenismo desempenham importantes papéis, visto que ocorrem alterações fisiológicas e anatômicas, dentre as quais, a diminuição na densidade do colágeno e das fibras musculares. Dentre os tratamentos não invasivos, a estimulação elétrica, método utilizado neste estudo, é um tratamento bem conhecido na clínica. Porém, raros são os trabalhos realacionados ao mecanismo de ação desse método terapêutico.

Sabe-se que, para a manutenção da continência urinária, é imprescindível a interação de alguns mecanismos, dentre esses, os que levam ao fechamento uretral. É importante lembrar que a resistência necessária para se obter a oclusão uretral é promovida, em parte, pela parede muscular da uretra ${ }^{8}$. A diminuição do tecido muscular, bem como o aumento do tecido conjuntivo entre os fascículos das fibras musculares, são referidos como fatores importantes da fisiopatologia da incontinência urinária ${ }^{9}$. Para a manutenção da continência urinária, os vasos periuretrais formariam uma espécie de corpo esponjoso, com função erétil, cuja pressão intravasal seria transmitida mecanicamente à uretra, obstruindo sua luz ${ }^{8}$.

Em um estudo no qual se avaliaram as estruturas uretrais de ratas castradas, foi demonstrado que a reposição estrogênica diminui significativamente a quantidade de fibras colágenas, com aumento significativo das fibras musculares, o que explica, em parte, a melhora da sintomatologia da incontinência urinária em mulheres quando da utilização da terapia hormonal ${ }^{10}$.

Dentre as técnicas para o tratamento clínico da incontinência urinária, a eletroestimulação funcional (FES) do assoalho pélvico tem sido utilizada tanto de forma ambulatorial quanto domiciliar, com resultados otimistas na melhora dessa afecção ${ }^{11}$. A FES utilizada neste estudo foi baseada em parâmetros já estudados, pois, para a obtenção de sucesso terapêutico, a frequência de $50 \mathrm{~Hz}$ é consensual entre os pesquisadores ${ }^{5,6,11}$. Além disso, estudos experimentais em ratas também utilizam essa mesma técnica ${ }^{7}$.

Para a incontinência de esforço, frequências iguais ou superiores a $50 \mathrm{~Hz}$ conseguem ativar o reflexo pudendo. Para a incontinência de urgência, empregam-se frequências entre 5 e $10 \mathrm{~Hz}$, que ativam o reflexo pudendo inibitório do detrusor, levando ao término ou diminuição das contrações involuntárias desse músculo; a largura de pulso oscila de 1 a $5 \mathrm{~ms}^{12,13}$.

Estudos realizados em humanos, em músculos atrofiados após cirurgias ou de indivíduos sedentários, mostraram que a fisioterapia com a ES aumentou significativamente a proporção das fibras musculares e a força muscular durante uma contração voluntária máxima sustentada, o que apoia nossos dados em relação à musculatura uretral ${ }^{14,15}$. Já Tam et al. ${ }^{16}$, denervando parcialmente os músculos tibial anterior, gastrocnêmio e plantar em ratos, observaram que não houve melhora histologicamente significante das fibras musculares entre o grupo controle e os que fizeram exercícios ativos ou FES. Contudo, esses dados ainda despertam controvérsias quanto a essa função reabilitadora na incontinência urinária. Neste trabalho, entre os parâmetros estudados, observamos aumento significante somente das fibras musculares periuretrais no grupo que recebeu 12 estímulos, ou seja, durante 3 semanas, o que nos leva a pensar que, em tecido saudável, é necessário um número maior e adequado de sessões para se obter a hipertrofia e consequente aumento de força muscular.

Além disso, a espera de uma semana para o sacrifício dessas ratas poderá ser o fator causal para o grupo de 3 semanas (6 estímulos) não apresentar hipertrofia, pois é sabido que, durante esse intervalo sem estímulos de contração, a musculatura poderá apresentar um retorno a sua condição inicial, o que talvez não tenha ocorrido com o grupo de 12 estímulos, uma vez que esse teve o dobro de sessões em relação ao anterior.

Com relação aos dados das concentrações de fibras colágenas, vasos sanguíneos e espessura do epitélio luminal analisados em nosso estudo, não verificamos diferenças significantes entre os grupos analisados. Porém, outros estudos mostraram haver relação entre o conteúdo de colágeno periuretral e a incidência de incontinência urinária ou distopias genitais. Alguns autores relatam que a quantidade de colágeno tipos I e III está significativamente reduzida ao redor da uretra e na fáscia pubocervical em mulheres com incontinência urinária de esforço em comparação às mulheres continentes ${ }^{17,18}$. Em contrapartida, outros pesquisadores relatam ter encontrado que a concentração de colágeno parece ser maior nas mulheres com incontinência urinária de esforço ${ }^{19,20}$ e nas mulheres que apresentam prolapso uterino $^{21}$, com maior identificação do colágeno tipo III.

Embora tenham sido realizados inúmeros estudos clínicos e experimentais, a fisiologia do tratamento clínico da incontinência urinária ainda está longe de ser plenamente elucidada, pois ainda faltam dados anatomofuncionais das estruturas do assoalho pélvico.

Assim, nosso estudo avaliou, por meio de um modelo experimental, os efeitos da FES do assoalho pélvico nas estruturas que compõem a uretra (epitélio urinário, colágeno, vasos e musculatura periuretral), onde se pode observar hipertrofia das células musculares lisas. Esta pesquisa acrescenta novas informações sobre a terapêutica da incontinência urinária pela eletroterapia; porém, surge aqui a necessidade de novas pesquisas, com maior tempo e número de sessões, para dar mais sustentabilidade aos dados encontrados, principalmente em relação ao tecido conjuntivo.

\section{Conclusão}

A eletroestimulação funcional prolongada do assoalho pélvico induziu aumento da camada muscular periuretral. 
1. Buckley BS, Lapitan MC; Epidemiology Committee of the Fourth International Consultation on Incontinence, Paris, 2008. Prevalence of urinary incontinence in men, women, and children. Current evidence: findings of the Fourth International Consultation on Incontinence. Urology. 2010;76(2):265-70.

2. Menezes GM, Pinto FJ, da Silva FA, de Castro ME, de Medeiros CR. [Complaint of urinary loss: a silent woman's problem]. Rev Gaucha Enferm. 2012;33(1):100-8.

3. Pereira VS, de Melo MV, Correia GN, Driusso P. Long-term effects of pelvic floor muscle training with vaginal cone in post-menopausal women with urinary incontinence: a randomized controlled trial. Neurourol Urodyn. 2013;32(1):48-52.

4. Knorst MR, Cavazzotto K, Henrique M, Resende TL. Physical therapy intervention in women with urinary incontinence associated with pelvic organ prolapse. Rev Bras Fisioter. 2012;16(2):102-7.

5. Castro RA, Girão M, Arruda RM, Takano CC, Sartori MG, Martins $S R$, et al. Does electrical stimulation of the pelvic floor make any change in urodynamic parameters? When to expect a cure and improvement in women with stress urinary incontinence? Clin Exp Obstet Gynecol. 2004;31(4)274-8.

6. Castro RA, Arruda RM, Zanetti MR, Santos PD, Sartori MG, Girão M. Single-blind, randomized, controlled trial of pelvic floor muscle training, electrical stimulation, vaginal cones, and no active treatment in the management of stress urinary incontinence. Clinics (São Paulo). 2008;63(4):465-72.

7. Franco GR, Oliveira Ed, Baracat EC, Simões M J, Sartoria $M G$, Girão MJ, et al. Histomorphometric analysis of a rat bladder after electrical stimulation. Rev Assoc Med Bras. $2011 ; 57(1): 20-4$.

8. Rud T, Andersson KE, Asmussen M, Hunting A, Ulmsten U. Factors maintaining the intraurethral pressure in women. Invest Urol. 1980;17(4):343-7.

9. Fitzgerald MP, Russell B, Hale D, Benson JT, Brubaker L. Ultrastructure of detrusor and urethral smooth muscle in women with urinary incontinence. Am J Obstet Gynecol. 2000; 182(4):879-84.

10. Sartori MG, Girão $M$, de Jesus Simões $M$, Sartori JP, Baracat EC, Rodrigues de Lima G. Quantitative evaluation of collagen and muscle fibers in the lower urinary tract of castrated and under-hormone replacement female rats. Clin Exp Obstet Gynecol. $2001 ; 28(2): 92-6$.
11. Santos PF, Oliveira E, Zanetti MR, Arruda RM, Sartori MG, Girão $M$, et al. [Electrical stimulation of the pelvic floor versus vaginal cone therapy for the treatment of stress urinary incontinence]. Rev Bras Ginecol Obstet. 2009;31 (9):447-52.

12. Amaro JL, Gameiro MO, Kawano PR, Padovani CR. Intravaginal electrical stimulation: a randomized, double-blind study on the treatment of mixed urinary incontinence. Acta Obstet Gynecol Scand. 2006;85(5):619-22.

13. Pena Outeiriño JM, Rodríguez Pérez AJ, Villodres Duarte A, Mármol Navarro S, Lozano Blasco JM. [Treatment of the dysfunction of the pelvic floor]. Actas Urol Esp. 2007;31 (7):719-31.

14. Valli P, Boldrini L, Bianchedi D, Brizzi G, Miserocchi G. Effect of low intensity electrical stimulation on quadriceps muscle voluntary maximal strength. J Sports Med Phys Fitness. 2002;42(4): 425-30.

15. Marqueste T, Hug F, Decherchi $P$, Jammes Y. Changes in neuromuscular function after training by functional electrical stimulation. Muscle Nerve. 2003;28(2):181-8.

16. Tam SL, Archibald V, Jassar B, Tyreman N, Gordon T. Increased neuromuscular activity reduces sprouting in partially denervated muscles. J Neurosci. $2001 ; 21$ (2):654-67.

17. Takano CC, Girão M, Sartori MG, Castro RA, Arruda RM, Simões $M$, et al. Analysis of collagen in parametrium and vaginal apex of women with and without uterine prolapse. Int Urogynecol J Pelvic Floor Dysfunct. 2002;13(6):342-5.

18. Goepel C, Hefler L, Methfessel HD, Koelbl H. Periurethral connective tissue status of postmenopausal women with genital prolapse with and without stress incontinence. Acta Obstet Gynecol Scand. 2003;82(7):659-64.

19. Bergmam A, Elia G, Cheung D, Perelman N, Nimni ME. Biochemical composition of collagen in continent and stress urinary incontinent women. Gynecol Obstet Invest. 1994;37(1):48-51.

20. Falconer $C$, Blomgren $B$, Johansson $O$, Ulmsten $U$, Malmström $A$, Westergren-Thorsson $G$, et al. Different organization of collagen fibrils in stress-incontinent women of fertile age. Acta Obstet Gynecol Scand. 1998;77(1):87-94.

21. Ewies AA, Al-Azzawi F, Thompson J. Changes in extracellular matrix proteins in the cardinal ligaments of post-menopausal women with or without prolapse: a computerized immunohistomorphometric analysis. Hum Reprod. 2003;18(10):2189-95. 